\title{
Current Scene and Prospective Potentials of siRNA in Cancer Therapy
}

\section{Mahavir B Chougule* and Rakesh K Tekade}

Department of Pharmaceutical Sciences, College of Pharmacy, University of Hawaii at Hilo, 96720, Hawaii, USA

Cancer is one of primary cause of death worldwide and its threat is estimated to touch 13.1 million human casualties in 2030 [1]. This disease occurs in all patient population i.e. young, old, children, men and women. As a whole, it represents a tremendous burden on patients, families and societies; and that's why needs urgent attention.

\section{Cancer Treatment Modalities: Position of siRNA}

Among currently available cancer treatment modalities- radiation, surgery, and chemotherapy have made momentous progress, but they have ample of limitations and are distant from ideal one [2]. For these rationales an effective, safe and patient-acceptable cancer treatment strategy is still largely an unmet goal. Recent understanding of the genetic basis of the disease opened the prospective for cancer gene therapeutics based on small interfering RNA (siRNA) as an alternative approach for cancer therapy. After introduction of this path breaking innovation, pharmaceutical companies and researchers throughout the globe have dedicated massive time and funds into the design of delivery system that can mediate safe and effective delivery of siRNA at target site. A striking feature of siRNA-based therapies is their potential to silence the expression of any disease-related gene in a selective and sequence-dependent manner [3]. This competence to target any transcribed genomic sequence has already made siRNAbased approaches an invaluable tool in validating novel targets in cellbased disease models.

RNA interference has already been observed in most organisms, from plants to vertebrates. This tactic is capable of providing new therapeutic modality for treating cancer, neurodegenerative diseases, antiviral diseases, Huntington's disease, hematological diseases, inherited genetic disorders, and many other illnesses [4]. With the established track record of silencing of important disease targets, the extension of siRNA technology as a therapeutic seems appealing.

\section{Benefits of siRNA over Drug Molecules}

The key advantage of siRNA over drug molecule lies in their ease of synthesis as well as low production costs as compared to other protein or antibodies. Available data suggests that siRNA has capability for their deliverance to a wide array of organs [5]. Another key factor is that the sequences can be rapidly designed for highly specific inhibition of the target of interest. Also, the synthesis of siRNAs does not require a cellular expression system, complex protein purification, and is relatively simple [6]. In spite of this, even after over a decade of exploration, a PubMed search for "siRNA" reflects over 45186 references with around 3000 on reports on its delivery approaches. However, until now only a handful of delivery approaches have been successfully transformed to the clinical level.

\section{In-Hand Hurdles With Naked siRNA Delivery}

Even though the biomedical potentials of siRNA are exceedingly high, there are some disputes that are hampering their practical applications. Because of their large molecular weight (MW $\approx 13 \mathrm{kDa})$, polyanionic and hydrophilic nature, they stumble upon the problem to enter cells by passive mechanisms. Endosomal trapping may sometimes results into null effect. Other major confronts in siRNA therapy are the prospective changes of getting active "off-target" as well as inducing the immune response [7]. After siRNA is internalized inside the cell, it must be released from the endosome to cytoplasm while avoiding entrapment and degradation. The in vivo siRNA delivery represents yet another gigantic challenge due to renal elimination as well as swift enzymatic digestion in plasma [8]. For example, naked siRNA has a half-life of less than $5 \mathrm{~min}$ in plasma [9]. Upon systemic administration, siRNA also suffer from nonspecific uptake by Reticulo Endothelial System (RES).

An in-hand available approach to improve siRNA's nuclease stability and pharmacokinetic profile is to directly modify its internucleotide phosphate linkage (by replacement of non-bridging oxygen with sulfur, boranophosphate, phosphoramidate or methyl groups). However extensive modification of a siRNA generally results in decreased activity. For instance, heavy modification with 2'O methyl can reduce potency or completely inactivate a siRNA [9]. There is no unique "or we can say best" modification methodology one may recommend for siRNA strand modification; rather it needs a judicious rationale base selection. Initially immense success was observed with viral vectors for delivery of siRNA that enabling efficient transduction efficiency, tissue-specific, and prolonged gene silencing [10]. Nonetheless, biosafety concerns mainly including host immune responses as well as mutagenesis restricted their clinical application [10]. Along with this, high-titer concentrations may infect many cells; and the higher chances of experiencing viral toxicity as well as occurrence of strong host responses resulting from the activation of the human immune system do exist there. Consequently, non-viral vectors came into picture towards development of as safe and effective alternatives. Although, siRNA can most successfully introduced inside cells employing electroporation or commercially accessible cationic lipid based vectors, but these strategies are not that successful owing to their restricted local application (with electroporation) or non-specific effects for lipid based vectors.

\section{Current Nanotech Alternatives for siRNA Delivery}

Mainstream of currently investigated non-viral siRNA delivery tactics are relying on complexation to safeguard the siRNA from the enzyme (RNase) rich in vivo environment as well as help siRNA transverse across the biomembranes. Regrettably, nanoparticle

*Corresponding author: Mahavir B Chougule, Department of Pharmaceutical Sciences, College of Pharmacy, University of Hawaii at Hilo, 96720, Hawaii, USA, Tel. +1 808-933-2906; Fax: +1 808 933-2974; E-mail: mahavir@hawaii.edu, mahavirchougule@gmail.com

Received September 13, 2012; Accepted September 14, 2012; Published September 19, 2012

Citation: Chougule MB, Tekade RK (2012) Current Scene and Prospective Potentials of siRNA in Cancer Therapy. J Pharmacogenom Pharmacoproteomics 3: e125. doi:10.4172/2153-0645.1000e125

Copyright: (c) 2012 Chougule MB, et al. This is an open-access article distributed under the terms of the Creative Commons Attribution License, which permits unrestricted use, distribution, and reproduction in any medium, provided the original author and source are credited. 
deliverance has been observed to bear limitations owing to inadequate bio distribution, rapid plasma clearance, low transfection efficiency, and sometimes associated cellular toxicities too [11].

Quite a lot of other synthetic designed vectors have been introduced for improving gene silencing applications. These chiefly includes: liposome, nano particles, microcapsules, cationic lipids, cationic polymers, cationic cell-penetrating peptides, dendrimers, and carbon nanotubes [12]. Still no perfect nanoscaled delivery system had been introduced, which fulfills all the prerequisites. Each of the current methods of gene delivery bears one or the other limitations and none fulfills the expectations completely. Many groups are searching for an optimal delivery tool that can be systemically administered, safe, and will deliver the siRNA specifically and efficiently to the target tissue.

\section{Expectations from Ideal siRNA Delivery Vectors}

With such limitations with siRNA delivery one may think- what makes for an ideal nano-vector? The ideal delivery device must be capable of (i) nanometric size range, (ii) biocompatible and nonimmunogenic, (iii) protecting siRNA from serum nucleases, (iv) prolong the blood retention time (v) avoid renal clearance, (vi) mediate effective bio distribution, (vii) mediate siRNA delivery into target cells while sparing normal tissues.

\section{On-going Clinical Trials with siRNA}

Currently, several biotechnology companies are trying to fetch clinical applications of siRNA. Most clinical trials employ deliverance of siRNA directly to the targeted tissue by local administration (for instance eye). On the contrary, for the treatment of cancerous mass, which is not freely reachable, systemic administration is vital. The foremost siRNA assessed in a human clinical trial setting was vascular endothelial growth factor-targeted siRNA for the treatment of macular degeneration [13]. Compared to earlier clinical trials, which mainly relied on local administration to easily accessible tissues, recent move is to treat inaccessible solid tumors via systemic administration was made by Calando Pharmaceuticals Inc. and Silence Therapeutics.

Tekmira Pharmaceuticals Corporation initiated a Phase I human clinical trial (TKM-PLK1) to establish safety and identify the maximum tolerated dose in relapsed or refractory cancer patients (www. tekmirapharm.com). Another Phase I clinical trial (Atu027) has been announced by Silence Therapeutics to treat broad range of solid tumors of liver, lung, prostate, melanoma, liver and others (www.silencetherapeutics.com). Atu027, a chemically modified siRNA formulated in liposomes that may result in a reduction in nutrient and oxygen supply to solid tumors [14]. A promising clinical trial employing targeting ligand based therapeutic is the RONDEL ${ }^{\mathrm{TM}}$ technology developed by Calando Pharmaceuticals. This cyclodextrin-based system containing anti-RRM2 (M2 subunit of ribonucleotidereductase) siRNA (commercially termed CALAA-01), has reached phase I clinical trials in the treatment of solid tumors. It was announced recently that in addition to CALAA-01, preclinical development of another siRNA oncology therapeutic is underway (www.calandopharma.com). These works provide proof of concept for non-viral targeted delivery of siRNA as a cancer therapeutic and illustrates the potential for further innovative delivery approaches.

\section{Toxicological Profiling, Scaling and GMP Compliance}

Stirring of current literature infers plentiful of in vitro and in vivo studies are trying to shed light on the toxicological profile of those innovative delivery systems, however in absence of systematic comparison and due to different protocols, cell lines, assays and in vivo models, the results are often inconsistent and controversial. Furthermore, scaling-up and GMP requirements for excipients as well as manufacturing protocols are hardly ever taken into consideration when novel delivery technologies are being investigated. However, since the field of siRNA is still relatively young, the number of ongoing clinical trials and also booming preclinical in vivo studies nevertheless promises a therapeutic as well as commercial potential for these molecule. To make the application of therapeutic siRNA a actuality, upcoming research must go on to focus on achieving resourceful delivery to the desired cells, minimizing off-target effects, increasing resistance to nuclease degradation, evading immune responses, and catching of polymerized particles by Kuffer cell and lung macrophages.

\section{Conclusion and Future Expectations}

The design and engineering of siRNA carriers gained note worthy impetus in recent years, as a result of buildup of predictable and therapeutically promising molecular targets. It is deeply anticipated that momentous progress in siRNA formulation development shall continue to enlighten to apprehend its possible therapeutic application. The future research has to focus on achieving well-organized delivery of siRNA to the desired cells (with no off-target effects), increasing resistance to nuclease, avoiding immune responses, trapping of polymerized particles by Kuffer cell and lung macrophage.

Development of siRNA formulation with excipients for long-term storage that do not require additional lyoprotectants/excipients for extending shelf-life will offer more ease for clinical use. Multifunctional siRNA carriers can circumvent many existing barriers by evading immune responses and prolonging circulation in the blood, achieving targeted delivery and facilitating efficient intracellular trafficking. Additionally, the siRNA that will be administered should have a highly specific sequence and possibly be modified chemically to attain greater levels of potency (i.e. reducing off-target effects and improving stability in serum). The pharmaceutical formulation scientists have to take gigantic strides to create a diverse array of functional carriers that can assemble siRNA in supramolecular complexes. An exhaustive comparison is also urgently desired among the available carriers to understand their relative performances and identifying the carrier with optimum potency.

The recent studies pursued supramolecular complexes from tailored carriers, siRNA and conventional small molecular drugs such as doxorubicin. The initial paradigm of siRNA therapy inherently assumed a single target for silencing. However, pathophysiological changes in tissues often result from changes in multiple targets. The combined effect of siRNA along with chemotherapeutic drugs, receptor up-regulation as well as blockade of drug efflux pump should be performed in exhaustive fashion. Independent studies have overwhelmingly demonstrated the feasibility of siRNA-mediated down-regulation using both non-viral and viral vectors, but complete knockdown is rare. What happens to sub-populations of cells where the molecular target is not silenced is an open issue in the literature. Will those cells display selective resistance to therapy and take over the pathophysiology, ultimately creating a phenotype resistant to the therapy? A systematic studies focusing on reasons for lack of complete down regulation will be needed to better understand this issue.

In addition, other understudied areas on this facade needs complete answer regarding (i) intracellular dissociation of engineered complexes, 
Citation: Chougule MB, Tekade RK (2012) Current Scene and Prospective Potentials of siRNA in Cancer Therapy. J Pharmacogenom Pharmacoproteomics 3:e125. doi:10.4172/2153-0645.1000e125

Page 3 of 3

and (ii) its bio-fate. Approaches to lower the toxicity without affecting bio-efficacy must be an added arena of exploration. In this line, PEGylation represents prototypical scheme. Bioconjugation of siRNA strands with lipids, polymers, or other biodegradable polymers can tremendously enhance the efficiency as well as uptake fate of siRNA. This strategy is not only capable of raising the thermodynamic stability of siRNA but also perk up its pharmacokinetic profile. Carriers or complexes with minimal cell interactions are also attractive, but their efficacy needs to be somehow enhanced by target specific ligand anchoring (folate, LHRH, Dextran, galactose). A systematic approach on suitable carrier design and performance testing is most likely to amplify the reassure level with clinical use of non-viral carriers, ultimately providing more opportunities for successful therapeutic use of the siRNA's. At the end, what we require is to work hard on the subject matter and anticipate the best to come!

\section{References}

1. http://www.who.int/cancer/en/

2. Tekade RK, Kumar, PV, Jain, NK (2009) Dendrimers in Oncology: An Expanding Horizon. Chem Rev 109: 49-87.

3. Bora RS, Gupta D, Mukkur TK, Saini KS (2012) RNA interference therapeutics for cancer: challenges and opportunities. Mol Med Report 6: 9-15.

4. Gavrilov K, Saltzman WM (2012) Therapeutic siRNA: principles, challenges, and strategies. Yale J Biol Med 85: 187-200.
5. Braasch DA, Paroo Z, Constantinescu A, Ren G, Oz OK (2004) Biodistribution of phosphodiester and phosphorothioate SiRNA. Bioorg Med Chem Lett 14 1139-1143.

6. Sah DW (2006) Therapeutic potential of RNA interference for neurological disorders. Life Sci 79: 1773-1780.

7. Jackson AL, Burchard J, Leake D, Reynolds A, Schelter J, et al. (2006) Position specific chemical modification of siRNAs reduces "off-target" transcrip silencing. RNA 12: 1197-1205.

8. White PJ (2008) Barriers to successful delivery of short interfering RNA afte systemic administration. Clin Experim Pharm \& Physiol 35: 1371-1376.

9. Behlke MA (2008) Chemical modification of siRNAs for In Vivo Use. Oligonucleotides 18: 305-320.

10. Tomanin R, Scarpa M (2004) Why do we need new gene therapy viral vectors? Characteristics, limitations and future perspectives of viral vector transduction. Curr Gene Ther 4: 357-372

11. Meade BR, Dowdy SF (2007) Exogenous siRNA delivery using peptide transduction domains/cell penetrating peptides. Adv Drug Deliv Rev 59: 134 140 .

12. Akhtar S, Benter IF (2007) Nonviral delivery of synthetic siRNAs in vivo. J Clin Invest 117: 3623-3632.

13. Ni Z, Hui $P$ (2009) Emerging pharmacologic therapies for wet age-related macular degeneration. Ophthalmologica 223: 401-410.

14. Strumberg D, Schultheis B, Traugott U, Vank C, Santel A et al (2012) Phase clinical development of Atu027, a siRNA formulation targeting PKN3 in patients with advanced solid tumors. Int J Clin Pharmacol Ther 50: 76-78. 